\title{
Intraarterial therapy for middle cerebral artery dissection with intramural hematoma detection on susceptibility- weighted imaging
}

\section{Journal of Neurocritical}

Changhyo Yoon, $\mathrm{MD}^{1}$; Seunguk Jung, $\mathrm{MD}^{1,2}$; Heejeong Jeong, MD, $\mathrm{PhD}^{1}$; Eunbin Cho, MD, PhD ${ }^{1,2}$; Tae-Won Yang, MD'; Seung Joo Kim, MD'; Ki-Jong Park, MD, PhD ${ }^{1,2}$; Seung Soo Kim, MD ; Hyun Park, MD, PhD ${ }^{3}$

${ }^{1}$ Department of Neurology, Gyeongsang National University Changwon Hospital, Changwon, Republic of Korea

${ }^{2}$ Department of Neurology and Institute of Health Science, Gyeongsang National University School of Medicine, Jinju, Republic of Korea

${ }^{3}$ Department of Neurosurgery, Gyeongsang National University Changwon Hospital, Changwon, Republic of Korea

\section{CASE REPORT}

Received: October 14, 2019

Revised: November 25, 2019

Accepted: November 29, 2019

Corresponding Author:

Seunguk Jung, MD

Department of Neurology, Gyeongsang National University Changwon

Hospital, 11 Samjeongja-ro, Seongsangu, Changwon 51472, Republic of Korea

Tel: +82-55-214-3814

Fax: +82-55-214-2638

E-mail: seunguk1358@gmail.com

Background: Intracranial artery dissection (IAD) may be an underdiagnosed cause of large vessel occlusion. The safety and efficacy of intra-arterial therapy (IAT) in patients with IAD are largely unknown. We report the case of a patient with IAD who was successfully treated with IAT.

Case Report: A 27-year-old man with a sudden-onset sensory dominant aphasia was admitted to our hospital around 16 hours after disease onset. Brain magnetic resonance angiography revealed an occlusion in the left distal middle cerebral artery (MCA). On the susceptibility-weighted imaging, bead-shaped dark signals were observed in the left MCA bifurcation, and intramural hematoma was suspected. We performed thrombectomy and permanent stenting for the dissecting MCA occlusion and achieved complete recanalization. Conclusion: The IMH on susceptibility-weighted imaging led us to suspect that the large vessel occlusion was due to the IAD. Further research is needed to address the efficacy and safety of IAT in patients with IAD.

Keywords: Middle cerebral artery; Blood vessel dissection; Thrombectomy

\section{INTRODUCTION}

Spontaneous intracranial artery dissection (IAD) may be an underdiagnosed cause of acute ischemic stroke. IAD is more frequently reported in the Asian population [1]. The pathophysiology of IAD and the cause of its prevalence in the Asian population are largely unknown. The radiological diagnosis of IAD in patients with acute ischemic stroke is often challenging because of the small size of intracranial arteries, and the subtle and nonspecific radiological findings. The pathognomonic radiological findings of
$\mathrm{IAD}$ are intramural hematoma (IMH), intimal flap, and double lumen. In stenoocclusive lesions without the aforementioned radiological evidence, IAD is difficult to predict. Recent advances in intraarterial techniques and thrombectomy devices have led to a high rate of recanalization in patients with large vessel occlusion (LVO). Intraarterial therapy (IAT) has become an essential treatment for patients with acute LVO. However, IAT in patients with IAD could have a high risk of arterial rupture and subsequent subarachnoid hemorrhage. Therefore, identifying the etiology of the LVO before the procedure can be useful in the development of an

(C) 2019 The Korean Neurocritical Care Society

This is an Open Access article distributed under the terms of the Creative Commons Attribution Non-Commercial License (http://creativecommons.org/licenses/by-nc/4.0/) which permits unrestricted noncommercial use, distribution, and reproduction in any medium, provided the original work is properly cited. 
effective and safe treatment strategy.

We report a case of acute middle cerebral artery (MCA) occlusion suspected to have an arterial dissection etiology based on susceptibility-weighted imaging (SWI) findings. The condition was successfully treated with IAT.

\section{CASE REPORT}

A 27-year-old man with a sudden-onset sensory dominant aphasia was admitted to the emergency department (ED) at 3:07 PM on March 19, 2018. When he got up at 11:00 AM, he noticed his condition. His last known well time was 11:00 PM the previous night. Previously, he was healthy and did not have any head trauma. His family history was also unremarkable. He was a current smoker with a 7-pack-year smoking history and denied alcohol consumption. His weight, height, and body mass index were 72.1 $\mathrm{kg}, 178 \mathrm{~cm}$, and $22.76 \mathrm{~kg} / \mathrm{m}^{2}$, respectively. On neurological examination, he was alert but could not obey simple commands. $\mathrm{He}$ could say more than three sentences but with incoherent content.
We concluded that these symptoms were compatible with Wernicke's aphasia. His motor strength was normal, and he showed no Babinski's sign or ankle clonus. The baseline National Institutes of Health Stroke Scale (NIHSS) score was 6. The brain magnetic resonance imaging (MRI) performed at another hospital before his ED visit at our hospital revealed an acute cerebral infarction on diffusion-weighted imaging (Fig. 1A, 1B). Signs of hyperintense vessels were found on fluid-attenuated inversion recovery imaging, and an occlusion was found in the left distal MCA on magnetic resonance (MR) angiography (Fig. 1C, 1D). SWI revealed bead-shaped dark signals in the area of the bifurcation site of the left MCA (Fig. 2A-2D), and bright signal intensities were observed on the phase map (Fig. 2E-2H). We performed emergency multimodal computed tomography (CT, Philips Healthcare, Eindhoven, The Netherlands) that included perfusion maps. We decided to perform IAT because of a large mismatch between the core, defined as a cerebral blood volume of $<2 \mathrm{~mL} / 100 \mathrm{~g}$, and the penumbra, defined as a relative mean transit time of $>145 \%$ when compared with the contralateral side (Fig. 1E, 1F).

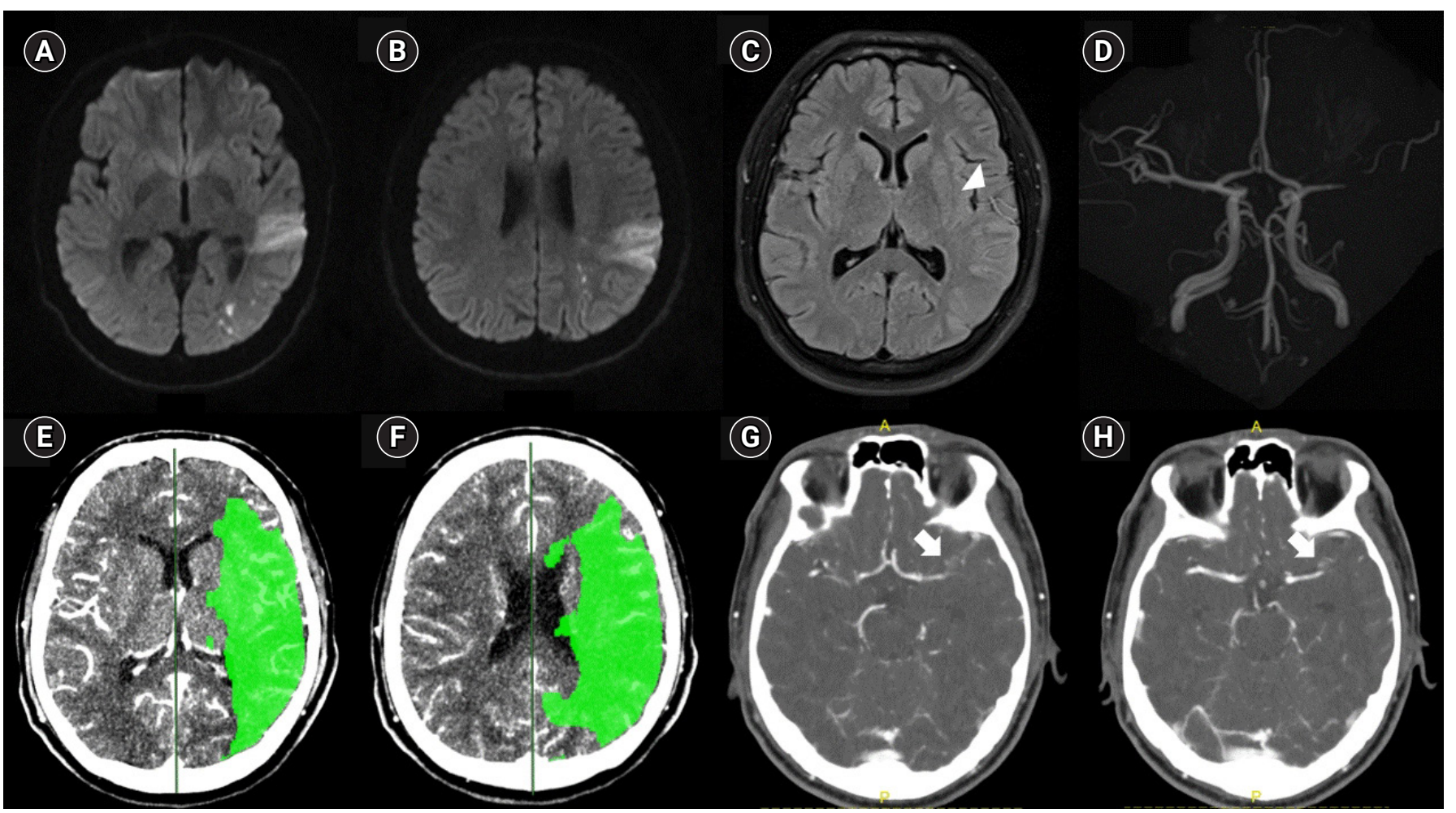

Fig. 1. Magnetic resonance (MR) imaging scans of the brain performed in another hospital before admission. (A, B) The diffusion-weighted image shows multiple infarctions in the left temporoparietal lobes. (C) Hyperintense vessel signs (white arrowhead) on fluid-attenuated inversion recovery imaging. (D) MR angiogram showing an occlusion in the left distal middle cerebral artery. ( $E, F)$ Perfusion computed tomography (CT) image showing perfusion delay, defined as a relative mean transit time of $>145 \%$ when compared with that on the contralateral side (green color), in the left middle cerebral arterial territory. No core infarct lesions, defined as a cerebral blood volume of $<2 \mathrm{~mL} / 100 \mathrm{~g}$ in the region on interest, were found. $(\mathrm{G}, \mathrm{H})$ Focal enlarged vessels with contrast enhancement (white arrows) on the CT angiography source images. 


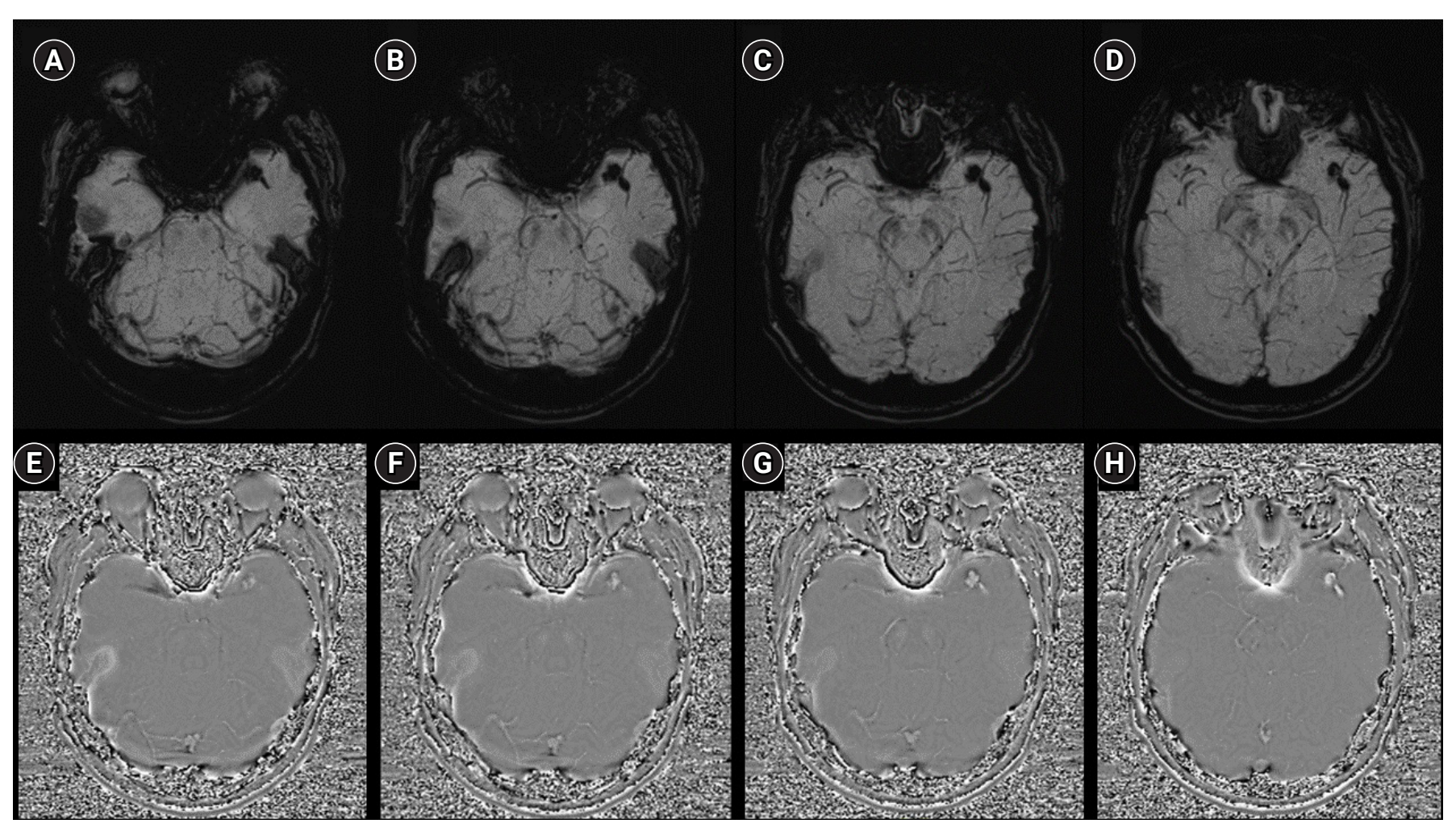

Fig. 2. (A-D) Susceptibility-weighted image demonstrating bead-shaped dark signals in the area of the bifurcation site of the left middle cerebral artery. $(\mathrm{E}-\mathrm{H})$ Corresponding bright signal intensities on the phase map.

Focal enlarged vessels with contrast enhancement were observed on the CT angiography source images (Fig. 1G, 1H). The initial blood pressure was $137 / 70 \mathrm{~mm} \mathrm{Hg}$, and the heart rate was 80 beats per minute. The results of the complete blood count, erythrocyte sedimentation rate, C-reactive protein level, urinalysis result, plasma electrolytes, and kidney, liver, and thyroid function tests were normal. The results of the serology tests for anti-Ro/ SSA, anti-La/SSB, antinuclear, antidouble-stranded DNA, antineutrophil cytoplasmic, and antiphospholipid antibodies were negative. The results of the human immunodeficiency virus, hepatitis B and C, and Venereal Disease Research Laboratory serology tests were also normal. The findings from the transthoracic echocardiography and 24-hour Holter examination were unremarkable.

A 9-Fr balloon guide catheter (Optimo, Tokai Medical Products, Kasugai, Japan) was introduced through a femoral sheath into the left carotid artery. A heparinized saline solution was continuously perfused through the catheter during the procedure. The initial internal carotid artery angiography revealed an occlusion in the left distal MCA (Fig. 3A, 3D). Thrombectomy was performed with the Trevo device (Stryker Neurovascular, Fremont, CA, USA), a retrievable self-expanding stent, and the left distal M1 and M2 inferior divisions were recanalized. After the thrombectomy, a filling defect in the left distal M1 and an occlusion in the left M2 superior division were found (Fig. 3B, 3E). Although we performed thrombectomy two more times for the recanalization of the left M2 superior division, recanalization was not achieved. The antegrade flow was restored when the stent was deployed, and we performed permanent stenting between the left distal M1 and M2 superior divisions by using a self-expandable stent $(3.0 \times 15$-mm Wingspan, Boston Scientific, Natick, MA, USA). Finally, we achieved complete recanalization of modified thrombolysis in cerebral infarction 3 (Fig. 3C, 3F). His neurological deficits recovered spontaneously. Both his NIHSS score at discharge and 90-day modified Rankin Scale score were 0.

\section{DISCUSSION}

To our knowledge, this is the first case of an MCA occlusion due to IAD, which was suspected, based on the IMH sign on SWI, and successfully treated with IAT. IMH is one of the pathognomonic radiological findings of IAD. IMH usually leads to an eccentric thickening of the arterial wall, with enlargement of the external diameter of the dissected artery. IMH can be observed as a hyperintense signal at 48 to 72 hours after onset on T1-weighted MRI. In several retrospective observational studies using conven- 


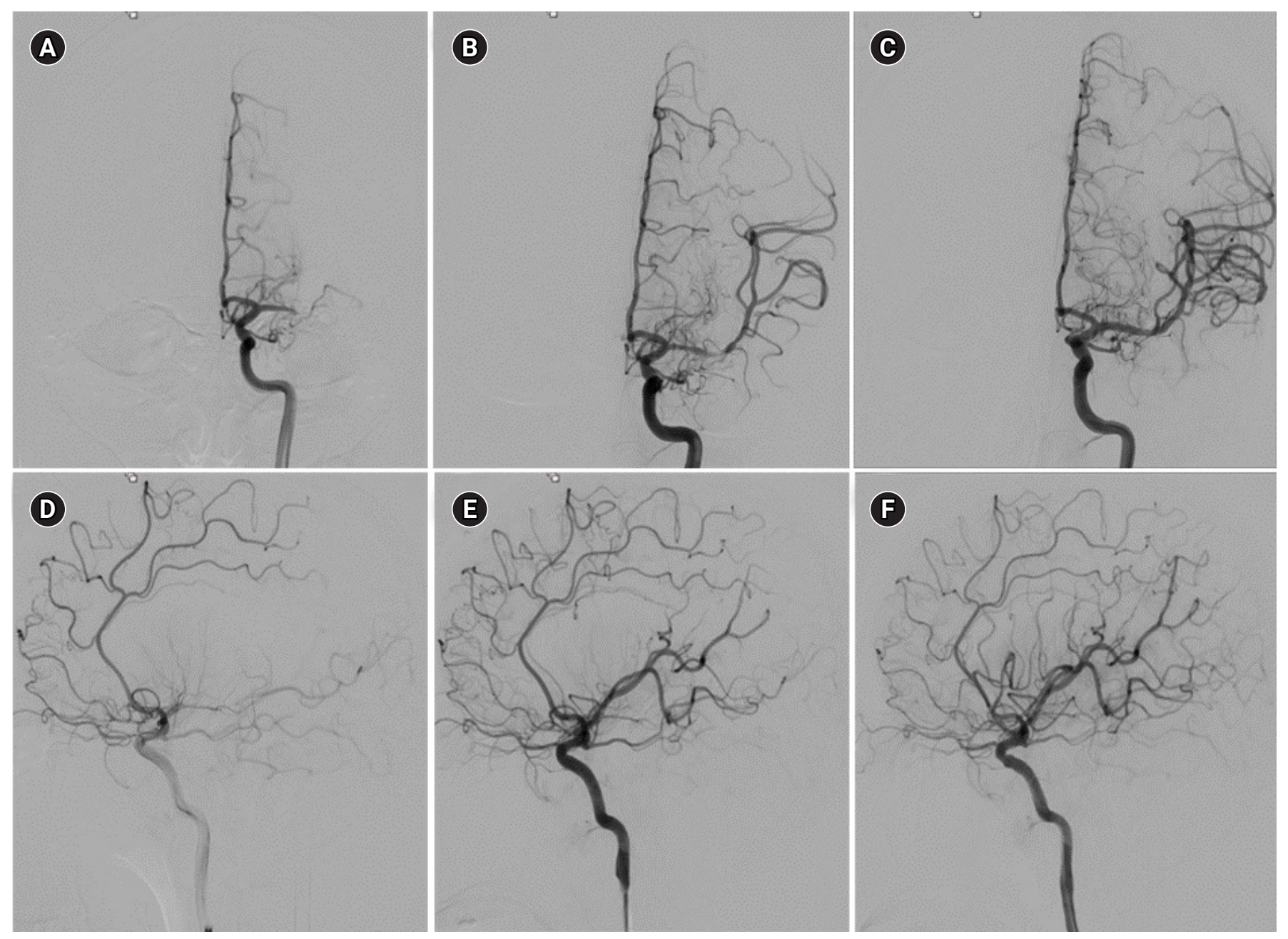

Fig. 3. Left internal carotid artery angiography (ICAG) images (A-C: frontal view; D-F: lateral view). ( $A$, D) The initial ICAG image shows an occlusion in the left distal middle cerebral artery (MCA). (B, E) After the thrombectomy using a stent retriever, a partial filling defect in the left distal M1 and an occlusion in the left M2 superior division were found. (C, F) Final angiogram after permanent stenting between the left distal M1 and M2 superior divisions, showing complete recanalization of the left MCA.

tional MRI, IMH was detected in only $32 \%$ to $34 \%$ of intracranial vertebrobasilar dissection cases $[2,3]$. The poor detection rate could be explained by the fact that IMH in the acute stage is rarely detectable on T1-weighted imaging because isointense hematomas are often obscured by surrounding tissue. IMT may be detected only in the subacute and early chronic stages owing to its paramagnetic effects [3]. Although the detection of IMH can be improved with high-resolution (HR) 3-T contrast-enhanced MRI or three-dimensional spin-echo T1 black-blood imaging, it also depends on the acquisition time from IAD occurrence $[4,5]$. However, the detection rate for dissections was higher with SWI than with the conventional MRI and may be less dependent on the MR acquisition time than any other MR modalities in terms of detection of hematomas $[3,6]$. In a retrospective study, IMH detected on SWI was positive in nine of 10 vertebral artery dissec- tions. The author showed that SWI had high sensitivity (90\%) and specificity (96.6\%) for detecting the IMH sign [7]. Among the patients, five underwent HR fat-suppression T1-weighted MRI. The eccentric high signal at the site of dissection, which was suggestive of IMH, was positive in only two of the five patients. The median time to the MRI studies was much longer in the patients with IMH (132 hours; range, 120 to 144) than in those without IMH on HR-MRI (10 hours; range, 5 to 16) [7]. SWI alone cannot differentiate IMH from calcification because it is sensitive to both paramagnetic and diamagnetic compounds. The phase map plays an important role in differentiating between hematoma and calcifications, as both have opposing signal intensities on the phase map [8]. In our case, the corresponding bright signals were observed in the phase map and were compatible with hematoma. Whether the IMH sign on SWI and T1-weighted MRI may 
also be seen in vulnerable atherosclerotic plaques with hemorrhage is unclear. Focal enlargement of the external diameter on T1-weighted imaging is associated with IMH [1]. In our case, enlarged vessels with contrast enhancement were observed on the $\mathrm{CT}$ angiography source images. Intraluminal clots in occlusive vessels may be seen as hypointense signals within the vascular cisterns on SWI and termed as the "susceptibility vessel sign (SVS)." IMH and SVS, especially in the occluded vessel, are difficult to differentiate on SWI. However, SVS is usually shown as a mass on SWI. In our case, the dark signals on the SWI had a bead-like appearance; therefore, IMH could develop in the occlusive spiral dissection. Spontaneous recanalization after stenting without clot retrieval also suggests arterial dissection rather than cardiac embolism.

Although IAT has become an essential treatment strategy in patients with acute LVO, its efficacy and safety in acute dissecting intracranial artery occlusion have not been evaluated, especially in the anterior circulation. Some observational studies have reported periprocedural hemorrhagic complications after endovascular treatment in $0 \%$ to $22 \%$ of patients with ischemic stroke from IAD [1]. If neurological symptoms are mild, it is better to avoid the procedure. If the procedure is inevitable because of disabling stroke, direct stenting after careful navigation through the true lumen might reduce the risk of periprocedural complications from repeated procedures. Therefore, identifying the etiology of the LVO before the procedure can be useful in the development of an effective and safe treatment strategy.

In conclusion, the IMH sign on SWI led us to suspect that the LVO was due to the IAD, and this finding is unaffected by the acquisition time from symptom onset. However, angiography may be necessary for the definite diagnosis of dissection because the reliability of the IMH sign on SWI has not been fully determined. This is needed to identify which features suggest IMH in arterial dissection rather than in intraplaque hemorrhage, and SVS in cardioembolic stroke. Further research is also needed to address the efficacy and safety in patients with disabling stroke from acute dissecting LVO.

\section{ARTICLE INFORMATION}

\section{Conflict of interest}

No potential conflict of interest relevant to this article.

\section{ORCID}

Changhyo Yoon, https://orcid.org/0000-0002-8875-6333

Seunguk Jung, http://orcid.org/0000-0003-2331-024X

Heejeong Jeong, https://orcid.org/0000-0001-6531-1860
Eunbin Cho, https://orcid.org/0000-0003-2021-8587

Tae-Won Yang, https://orcid.org/0000-0002-8113-2384

Seung Joo Kim, https://orcid.org/0000-0001-9391-1767

Ki-Jong Park, https://orcid.org/0000-0003-4391-6265

Seung Soo Kim, https://orcid.org/0000-0002-2114-4594

Hyun Park, https://orcid.org/0000-0002-2422-4389

\section{Author contributions}

Conceptualization: CY, SJ, SSK, and HP. Visualization \& Writing-original draft: CY and SJ. Writing-review editing: HJ, EC, TWY, SJK, and KJP.

\section{REFERENCES}

1. Debette S, Compter A, Labeyrie MA, Uyttenboogaart M, Metso TM, Majersik JJ, et al. Epidemiology, pathophysiology, diagnosis, and management of intracranial artery dissection. Lancet Neurol 2015;14:640-54.

2. Hosoya T, Adachi M, Yamaguchi K, Haku T, Kayama T, Kato T. Clinical and neuroradiological features of intracranial vertebrobasilar artery dissection. Stroke 1999;30:1083-90.

3. Ahn SS, Kim BM, Suh SH, Kim DJ, Kim DI, Shin YS, et al. Spontaneous symptomatic intracranial vertebrobasilar dissection: initial and follow-up imaging findings. Radiology 2012; 264:196-202.

4. Swartz RH, Bhuta SS, Farb RI, Agid R, Willinsky RA, Terbrugge $\mathrm{KG}$, et al. Intracranial arterial wall imaging using high-resolution 3-tesla contrast-enhanced MRI. Neurology 2009;72:62734.

5. Edjlali M, Roca P, Rabrait C, Naggara O, Oppenheim C. 3D fast spin-echo T1 black-blood imaging for the diagnosis of cervical artery dissection. AJNR Am J Neuroradiol 2013;34:E103-6.

6. Takano K, Yamashita S, Takemoto K, Inoue T, Kuwabara Y, Yoshimitsu K. MRI of intracranial vertebral artery dissection: evaluation of intramural haematoma using a black blood, variable-flip-angle 3D turbo spin-echo sequence. Neuroradiology 2013;55:845-51.

7. Kim TW, Choi HS, Koo J, Jung SL, Ahn KJ, Kim BS, et al. Intramural hematoma detection by susceptibility-weighted imaging in intracranial vertebral artery dissection. Cerebrovasc Dis 2013;36:292-8.

8. Schweser F, Deistung A, Lehr BW, Reichenbach JR. Differentiation between diamagnetic and paramagnetic cerebral lesions based on magnetic susceptibility mapping. Med Phys 2010; 37:5165-78. 